\title{
Ueber die Fähigkeit der Zelle, activ ihr specifisches Gewicht zu verändern.
}

\author{
Von \\ Max Verworn, \\ Dr. med., Privatdocent a. d. Universität Jena.
}

\section{Einleitung.}

Unter den Vertretern jener märchenhaften Thierwelt, welche die oberen Schichten der Meere bevölkert, ist bekanntlich eine grosse Zahl mit der wunderbaren Fähigkeit begabt, ohne Vermittelung der Locomotionsorgane im Wasser langsam auf und nieder zu schweben. Am weitesten verbreitet ist diese merkwürdige Erscheinung bei den Gruppen der Radiolarien, Ctenophoren and Siphon ophoren, aber noch bei manchen andereu pelagischen Thieren dürfte sie sich finden und schliesslich ist sie auch bei einigen $R \mathrm{~h}$ i z o pod e $n$ des suissen Wassers beobachtet worden.

Da man sich durch genaue Beobachtung in der That leicht überzeugen kann, dass bei diesem Steigen und Sinken keine Locomotionsorgane im Spiele sind, so könnte man auf die Vermuthung kommen, dass Differenzen im specifischen Gewicht des Meerwassers, die ja überall vorhanden sind, eine äussere Ursache dieser Erscheinung abgeben. Das specifische Gewicht des Meerwassers ändert sich, besonders in offenen Gefässen, schon in kurzer Zeit durch Verdunstung des Wassers. Ich habe öfter Ctenophoren, die einen Tag uber in einem offenen Gefäss mit Meerwasser gestanden hatten und auch bei vollkommener Ruhe der Locomotionsorgane an der Oberfläche lagen, in frisch geschöpftes Meerwasser von gleicher Temperatur gebracht. Die Thiere fielen dann stets langsam zu Boden und oft erst im Lauf einer Stunde war die Gewichtsdifferenz durch Diffusion ausgeglichen. Das entsprechende Resultat ergab der umgekehrte Versuch: Frisch ein- 
Ueber die Fähigkeit der Zelle, activ ilır specifisches Gewicht zu verändern. 141

gefangene Ctenophoren, die in Ruhe am Boden des Gefässes lagen, in abgestandenes Seewasser gebracht, stiegen hier in die Höhe und blieben an der Oberfläche liegen. Durch abstufbare Verdünnung des Meerwassers mit suissem Wasser konnte ich diese Versuche noch exacter gestalten. Jedenfalls werden solche äusseren Ursachen gelegentlich anch im Meere unter natïrlichen Bedingungen wirksam sein, z. B. in Strömungen, bei starken Regrengiissen ete. Aber man kam in der gröissten Mehrzahl der Fälle auch diese äusseren Ursachen ausschliessen. Hält man z. B. die Thiere in langsam fliessendem Vasser oder schittzt man die Gefässe ror Verdunstung, so kann man beobachten, dass in demselben Gefaiss ein Theil der Thiere bei Rube der Locomotionsorgane am Boden, ein anderer Theil dicht unter der Oberfläche liegt und dass sie immer wieder in diese Lage zurückkehren, so oft man sie daraus entfernt. Erst wenn man die Thiere lange Zeit, mehrere 'Tage lang' in Gefässen gehalten hat, liegen bei Ruhe stets alle Thiere am Boden und können sich nur activ durch Thätigkeit ihrer Locomotionsorgane erheben. Dagegen gleichen sich dic Gewichtsdifferenzen, welche bei Einbringen der Thiere in Wasser von anderem specifischem Gewicht vorhanden sind, wach meinen Erfahrungen immer schon in der kuzen Zeit von höchstens 2 Stunden vollkommen ans. Zahlreiche ahuliche Beobachtungen zeigen dentlich, dass in den meisten Fällen nicht die Verschiedenheit des specifischen Gewichts des Wassers der Grund für das Steigen und Sinken der Thiere sein kann.

Kann man einerseits die Thätigkeit der Loconotionsorgane und andererseits äussere Ursachen für das Zustandekommen des Steigens und Siukens ausschliessen, so bleibt nur noch die Möglichkeit, dass diese Erscheinung bedingt ist durch active Veränderung. des specitischen Gewichts der 'Thiere.

Wie kann ein Thiersein specifisches Gewicht activ verändern? Stellt man sich in einem Medium schwimmend einen lebendigen Organismus ror, der das gleiche specitische Gewicht hat wie das ihn umgebende Medium, so giebt es offenbar nur zwei Möglichkeiten, wie dieser Organismus sein specifisches Gewicht verändern kann, nämlich entweder, indem er in seinem Stoffwechsel 
Stoffe producirt, die specifisch schwerer oder specifisch leichter sind wie das Medium oder indem er aus dem Medium schwerere oder leichtere Stoffe in sich aufnimmt und anhäuft. Beide Fälle sind in der Natur verwirklicht.

Betrachten wir zunächsst den ersten Fall. Das Protoplasma ist in der Regel specifisch etwas schwerer als Wasser. Es wïrden also hier nur die Fälle in Betracht kommen, wo im Stoffwechsel Stoffe producirt werden, die leichter sind als Wasser, die das specifische Gewicht des Organismus vermindern. Viele Stoffe, welche normaler Weise im Protoplasma der Zelle producirt werden, sind bekanntlich leichter als Wasser, vor allem die Fette und Gase. Producirt also ein Organismus, der im Wasser am Boden liegt, viel Fett, so kann leicht sein specifisches Gewicht so vermindert werden, dass der Körper leichter wird wie Wasser und allmählich im Wasser in die Höhe steigt. Ob derartige Fälle bei der Locomotion der Organismen verwirklicht sind, ist meines Wissens nicht bekannt, aber sehr wohl möglich. Bei nicht im Wasser lebenden Organismen kann durch Fettbildung, durch Fettmetamorphose der Gewebe eine oft bedeutende Verringerung des specifischen Gewichts eintreten, wie z. B. beim Mensehen mit der Entwickelung eines starken Panniculus adiposus oder unter patbologisehen Verhältnissen in der Leber bei Phosphorvergiftung.

Bekannt dagegen ist bereits seit langer Zeit die Bedentung der Gasentwicklung für die Locomotion gewisser Organismen. Es giebt im Slisswasser kleine Rhizopodenformen, welche mit einer zierlichen, oft aus Sandkörnchen zusammengesetzten Schale bedeckt sind, aus deren Oeffnung der formwechselnde Protoplasmakörper seine Pseudopodien berausstreckt. Solche Formen, wie A r c ella und D iff $1 \mathrm{u} g$ i a leben am Grunde der Gewässer und kriechen zwischen allerlei Schlamm und Detritus mit ibren Pseudopodien umher. Isolirt man sie mit einem Capillarrohr aus dem Bodensatz und setzt sie in klares Wasser, so fallen sie zu Boden, sind also schwerer wie Wasser. Diese Organismen, die sich unter normalen Umständen stets am Boden aufhalten, können in gewissen Fällen in die Höhe steigen. Untersucht man solche an der Oberfläche schwimmende Individuen unter dem Mikroskop, so bemerkt man, dass sie eine oder mehrere Gasblasen in ihrem Protoplasma enthalten. Untergetaucht kehren sie immer wieder an die Oberfläche des Wassers zurück, sie sind jetzt also leichter 
Ueber die Fähigkeit dor Zelle, activ ihr specifisches Gewicht zu verändern. 143

als Wasser. Diese Gasentwicklung bei den Rhizopoden ist nicht selten $\mathrm{zu}$ beobachten und ist auch bereits mehrfach beschrieben worden, besonders cingchend von Enge Iman ${ }^{i}$ ) bei Arcella v ulgaris. Das. Gas, welches im Körper entwickelt wird, ist wahrscheinlich Kohlensäure, doch liegen genanere Untersuchungen dariber nicht vor. E n g e 1 ma n n giebt an, dass die Gasblasen vom Protoplasma wieder resorbirt werden können. Ich habe bei Difflugia noch einen anderen Modus kennen gelernt, wie die Gasblasen entiemt werden, namlich durch einfachen Austritt ans dem lrotoplasma und der Schaleniiffunne. In jodem Falle aber sinken die Protisten nach dem Verschwinden der Gasblasen wieder zu Boden.

Dieses active Steigen durch Gasentwicklung und das spätere Nicdersinken nach dem Verschwinden der Gasblasen kamn von „rosser hiologiseher Bedeutung fïr die Protisten werden, worauf der tolgende Fall, den ich im Jahre 1886 in Berlin beohachtete, hinweist. In einem grösseren Gefäs befanden sich eine ungehcure Menge ron D ifflug i a lobost o m a, die alle in Schlamm am Boden ihr Dasein führten. An der Oberflaiche des Gefässes zeigte sich kein einziges Individuum. Als das Gefüss einige Zeit gestanden hatte, begann das Wasser etwas zu riechen, da am Boden Fäuluissprocesse auftraten. Zn dieser Zeit begannen die D if f $l u g$ i e $n$ sämmtlich Gasblasen zu entwickeln und an dic Oberfläche zu steigen, wo sie in dichten Schaaren, jcde mit einer oder mehreren Gasblasen versehen, frei schwebten. Scbliesslich aber starben die D iffll u g i e n mach und nach in dem Gefiss ganz aus, wärend das Wasser weiter faulte. Wie ich noch bemerken will, habe ich die Gasentwicklung auch bei A r cell a inmer epidemisch in einer Cultur auftreten sehen, niemals sporadisch. Hieruach liegt es nahe anzunelmen, dass durch die eingetretenen Fäulnissprocesse der Stoffwechsel in den D i f f 1 u g i e n in gewisser Weise gestört wurde und dass die Gasblasenentwicklung auf diesen Grund zuriickzufïhren ist. Das ist wichtig, denn tritt in einem Gewässer an irgend einer Stelle des Grundes, wo D i f'f 1 ug i e n leben, eine Störung in den Lebensbedingungen der Protisten ein und entwickeln sich in Folge dessen Gasblasen in ihrem Protoplasma, so verlassen

1) T h. W. Fngelmann: "Briträge zur Physiologie des Protoplasma." In Pflügers Arch. Bil. 2. 1849\%. 
sie dadurch den gefählilichen Ort, erheben sich an die Oberfläche und werden nun von Wind und Wellen an eine andere Stelle verschleppt, wo sie nach den Austritt der Gasblasen zu Boden sinken und günstigere Lebensbedingungen finden.

Ausser den Stisswasser-Rhizopoden haben noch die wundervollen meerbewohnenden Siphonophoren die Fiahigkeit durch Production und Abgabe von Gasblasen im Meere activ zu steigen und zu sinken. Die Siphonophoren besitzen zu diesem Zweek eine bestimmt localisirte Luftkammer, doch sind die Einzelheiten der Gasproduction und Gasabgabe sowie der damit verbundenen Locomotion noch nicht eingeheuder untersucht worden.

Soviel uber die Veränderung des specifischen Gewichts durch Production gewisser Stoffe in Stoffwechsel. Bisher nicht bekannt war die zweite Art der Veränderung des specifischen Gewichts, die, welche durch Aufnahme specifisch schwererer oder leichterer Stoffe aus dem Medium her hedingt ist. Gerade diese Art aber ist bei den pelanischen Seethieren, besonders hei den Radiolarien und Ctenophoren schr verbreitet.

Unter den Zoo!ogen und Physiologen ist die Frage, wie die Ortsverändertugen dieser Thiere zu Stande kommen, wemn die Locomotionsorgane in vollkommener Ruhe sind, bereits meluffach erörtert worden und es sind zum Theil recht abentenerliche Anschaumgen dariiber aufgetaucht. So stellte sich z. B. E i m e r ${ }^{1}$ ) vor, dass der ron Wasser befreite Kïrper der Ctenophoren leichter sein miisste wie Wasser und wie ein trockener Schwamm an der Oberflïche des Wassers schwimmen würde. Schon Krukenb e $\mathbf{r}_{\mathrm{g}}{ }^{2}$ ) hat dem gegenuiber geltend gemacht, dass dies nur unter der Voranssetzung richtig wïre, wenn statt des Wassers Luft die Gewebe des Ctenophorenkörpers erfüllte. In diesem Ctenophorenkörper witrde nun, so stellt sich Eimer weiter vor, Was ser durch Adsorption verdicbtet, indem die feinen Ge-

1) G. Th. Eimer, ,Versuche über künstliche Theilbarkeit von Beroë ovatus. Angestellt zum Zweck der Controlle seiner morphologischen Befunde über das Nervensystem dieses Thieres." In Arch. f. mikr. Anatomie Bd. XVII, 1880.

2) C. Fr. W. Kruken berg, „Bemerkungen zu der Eimer'schen Ansicht über den Ortswechsel der Rippenquallen." In Vergl.-physiologische Studien. Albth. III, 1880 . 
webslïcken und. Spalten wie capillare Räume wirkten. Durch diese Verdichtung les Wassers würde der Körper schwerer wie Wasser und sinke in Wasser unter. Welche physikalische Ungeheutrichkeit dieser Auffassung zu Grunde liegt, wird jedem klar werlen, wenn er sich an die tiberans geringe Compressibilität des Wassers crinuert. Bekanntich hedart es cines Drucks ron mehr als 2(n) Atmophäiren, um Wasser auch nur um den hundertsten Theil seines Volumens zu verdichten! Dass man nach einem annähernd so grossen Druck in den weichen Tewchen des Ctenophorenkïpers wohl vergeblich suchen dïrfte, wird niemand erustlich in Ervägung ziehen. K r u $\mathrm{k}$ e $\mathrm{n}$ h e r g, der bereits diese Momente bei dor liritik ron Eimer's Vorstellung hervorhebt. calaubt nun, dass die Ctenophoren in derselben Weise wie dic Rhizuporlen dex Susswassers durch Production von Gasblasen in Meere in die Hiile steigen Indessen ist es leicht, sich davon zu iibcrzeugen, dass anch lei den Thieren, die ruhig an der Oberflache des Wasscrs seluweben, keine Spur ron Gasblasen im Gewebe zu tinden ist, so lass anch die krukenberg'sche Vermuthmg nicht mutreffend ist.

Als ich mich in Jahre 18s! längere Zoit zum Zwecke physiologischer Studion an Mittelmeer aufhielt. bin io h der Frage wie dieses Steigen und Sinken der pelagisehen Thiero zu stande kommt, experimentell näher getreten. Da die Veraindernug des specilisehen Geviehts eines Thieres eine Lebensersehrinung ist, so wird das Problem dort liegen; wo die Lebensprozesse im Gewebe sich abspielen, d. h. in der Zelle. Wie alle plyysiologischen Probleme wird also auch dieses in letatcr lustanz cellular zu hehandeh sein und sich auf die Frage zuspitzen: wic verïndert die Ctenophoren-oder liadiolar ienzelle actir ibr specifisehes Gewicht? Die Radiolarien als einzellige, freilebende Oronismen werden fiir diese cellularphysiologischen Untersuchungen die geeignetsten Objekto sein.

\section{Versuche.}

Thalassicolla nucleata ist ein erbsengrosses Radiolar, das bei oberfächlicher Betrachtung grosse Aehnlichkeit mit einem Froschei hat. Der kugelrunde Kïrper repraisentirt eine /elle mit einem einzigen Zellkern und besteht aus einer Anzahl concentrischer 
Schichten (Fig. 1). Im Mittelpunkt liegt die Centralkapsel (3), welche das grobkörnige intrakapsuläre Endoplasma mit seinem Zellkern umschliesst. Aussen ist die Centralkapsel umgeben von einer diunnen Lage soliden Protoplasmas, das eine grosse Masse schwarzblaner Pigmentkörnchen enthält, so dass die Centralkapsel als schwarze Kugel innerhalb des sonst ziemlich durchsichtigen Körpers erscheint. Die solide Protoplasmalage geht nach der Peripherie in eine sehr stark vacuolisirte Schicht von beträchtlicher Breite uber (2), deren Vacuolen ziemlich gross und von sehr dünnen Protoplasmawänden umschlossen sind, so dass die Vacuolenschicht ein schaumiges Aussehen besitzt. Die Vacuolenschicht ist nach aussen von einer dicken Gallertschicht (1) umgeben, durch welche die radiär vom Mittelpunkt ausstrahlenden Pseudopodien als direkte Fortsetzung des Vacuolenwandprotoplasmas frei ins Medium

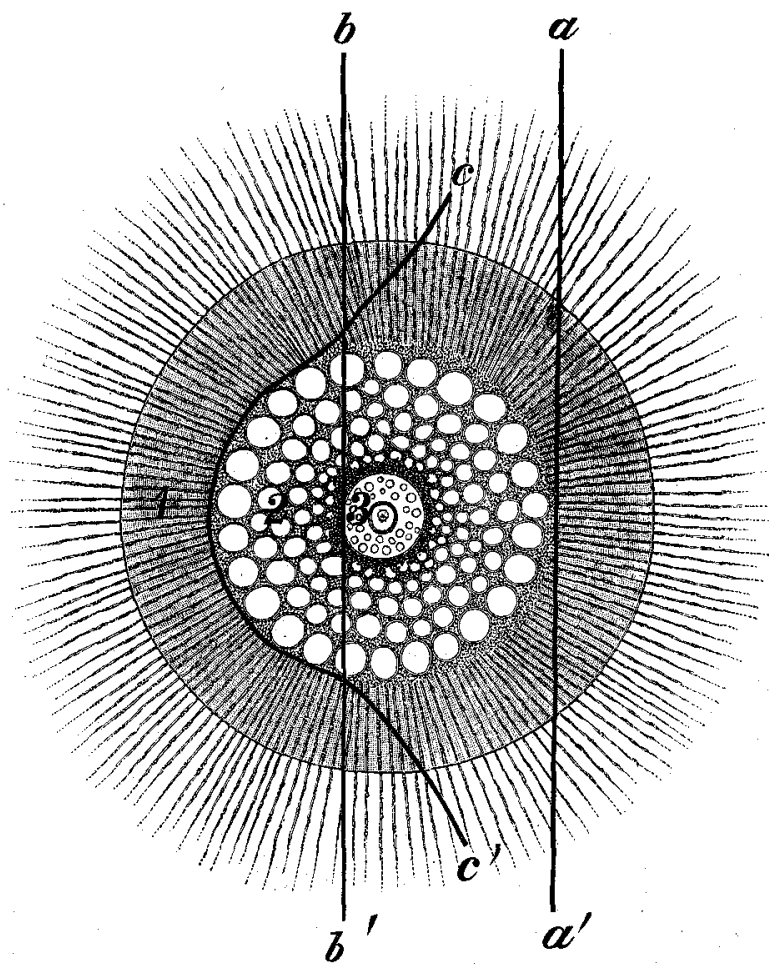

Fig. 1. Thalassicolla nucleata:

1. Gallertschicht. 2. Vacuolenschicht. 3. Centralkapsel mit Zellkern. Die Linien geben die Schnittrichtung bei den Operationen an. 
Úeber die Fähigkeit der Zelle, activ ihr specifisches Gewicht zu verändern. 147

hinausragen. Wegen der beträchtlichen Grösse des einzelligen Körpers und wegen der grossen Resistenzfähigkeit ist $\mathrm{T}$ halassic olla nucleata für vivisectorische cellularphysiologische Untersuchuugen eins der günstigsten Objecte, das man sich wiinschen kann.

Unter normalen Lebensbedingungen, weun die $\mathrm{T}$ h a $\mathrm{l}$ as s $\mathrm{i}^{-}$ collen ungestört in unbewegtem Wasser siud, schweben alle ausgewachsenen Individuen an der Obertäche des Wassers und ein spontanes Sinken habe ich nie bemerkt, ausser wenn sich au cinem Individum einmal ein Nahpungsorganismus getungen hatte, der es durch seine Schwere zu Bodeu rog. Werden die Protiston dagegen stark gereizt, etwa durch lange andauerndes heftiges Schitteln, oder durch chemische Reize, so sinken sie allmählich zu Boden, eive Erscheinung, die man noch besser bei der bedentend weicheren Tha las s i colla p elagi c a beobachten kann.

Fiu die Beantwortung der Frage: w i e $k$ a n n d i e $R$ a d $i$ olarienzelle ihr specifisches Gew icht activ veränd e rn, war es daher zunächst nothwendig festzustellen, welche Elemente der 'T halas i collen zelle in ungereizten Zustancle specifisch leichter sind wie das umgebende Meerwasser. Diese Fragc konnte ich sehr leicht durch operative Versuche entscheiden, indem ich vou jeder der eimzelnen Schichten systematisch Stiticke mit einem feinen Instrument abtrug und prifte, ob sie im Meerwasser untersanken oder an der Obertläche sehwehen blieben.

Die Centralkapsel aus dem Kirper herauszunehmen ist nioht sebwer. Man sticht mit einer schmalen Lanzette den Gallertkörper an und ibt dann einen mässigen Druck anf denselben aus, damn tritt, von Pigment befreit, die gelbe Centralkapsel unverletzt dureh den Stichkamal nach aussen. Lässt man die so isolirte Centralkapsel in ein Gefäss mit Meerwasser fallen, so sinkt sie darin schnell zu Boden und bleibt hier dauemd liegen. Die Centralkapsel ist also schwerer als Mcerwasser. Wurch eine ähnliche Operation kann man auch den Inlatt aus der Ceutralkapsel heraustreten lassen und findet dann, dass anch die einzelnen Bestandtheile des Inhalts, das intrakapsuläre Protoplasma und der bläschenfömige Zellkern sämmtlich schwerer sind wie Meerwasser. Der von der Centralkapsel befreite T h a las s i co ll e n körper schwimmt nach wie ror der Operation an der Oberfläche des Wassers und kehrt, wenn man ihn untertaucht, immer wieder da- 
hin zurück. Schnitt ich darauf von der kapsellosen Kugel mit einem feinen Schnitt $\left(a-a^{\prime}\right)$ ein Stick der Gallertschicht (rechts von der Linie a-a') ab, ohue daranhängende Vacuolen, so sank auch dieses zu Boden und blicb hicr liegen. bis es zerfiel. Schnitt ich dagegen (durch den Schnitt $b-b^{\circ}$ ) ein Stiick Gallertsubstan\% mit einem grösseren Theil der daranbïngenden Vacuolenschicht ab (links von $\mathbf{b}-\mathbf{b}^{\prime}$ ), so blieb diese Masse an der Oberfläche schweben. War die anbängrende Vacuolenmassc nur klein im Verbältniss zur abgeschnittenen Gallertnasse, so sank das Stiick zu Boden, aber bedeutend langsamer als vur ein Stück (tallertsubstanz allein. Scbliesslich schälte ich von einer $\mathrm{T}$ h a 1 as s i c olla die Gallertschicht $\left(c-c^{\prime}\right) a b$, und schnitt darauf von der darunterliegenden Vacuolenschicht ein Stiuck (zwischen $b-b^{\prime}$ und $c-c^{\prime}$ ) ab, so dass es nur Vacuolen und nichts von den übrigen Schichten enthielt. Diese Stücke blieben stets an der Oberfläche schweben und kehrten, wemn ich sie untertanchte, sehr schnell wieder an die Oberfläche zuriick. Damit war also klar bewiesen, dass die Vacuolenschicht das Element repräsentilt, welehes specifisch leichter ist als Meerwasser. Die beste Bestätigung hierfïr liegt in folgender Beobachtnng. Wenn nan eine Th a la s s i colla der Centralkapsel beraubt hat, gebt sie langrsam zu Grunde, indem die Gallertschicht allmählich sehleimig zerfliesst und die Vacuolen zerplatzen. Ist der grösste Theil der Vacuolen zerplatzt, so sinkt das Stiick zu Boden. Wenn man es nun in einen Uhrschälchen mit viel Wasser unter dem Mikroskop beobachtet und gelegentlich einmal stark schiittelt, so lösen sich häufig einzelne isolirte Vacuolen mit ringsherum geschlossener Wand von dem am Boden liegenden Stücke los und steigen dann an die Oberfläche, wo sie meist nach kurzer Zeit zerfallen, indem die vorber glatte Protoplasmawand rumzelig wird, anseinander geht und dem Meerwasser den Eintritt gestattet. Die Reste der zerfallenen Protoplasmawandschicht sinken dann wieder zu Boden. Diese Beobachtung zeigt nicht nur, dass jede Vacuole speeifisch leichter ist als Meerwasser, sondern sie beweist auch, dass das specifisch leichtere Element der Vacuolen in halt ist, während die Protoplasmawand der Vacnole schwerer ist als Meerwasser.

Nachdem so festgestellt war, dass die Vacuolen diejenigen Elemente sind, welche den Radiolarienkörper an der Oberfläche des Wassers schwebend erhalten, musste die weitere Frage entschieden werden, welches das Verlat tender Vacuolen 
ist, das die Erhöh ungdes specifischen Gewichts der Zelle und damit das Sinken beding t. Wie schon bemerkt, sinken die Thalassicollen nur unter, wenn sie andauernd stark gereizt werden, wie dies z. B. bei Stiimen auf dem Meere geschieht, nach denen of tagelang keine Radiolarien oder iiberhaupt pelawische Thiere an der Oberfläche des Wassers zu finden sind. Was geschieht bei der Reiznng der Thalassicolla mit den Vacuolen? Die Wirk ng aller Reize bei den Rhizopoden ist stets dieselbe: Das Protoplasma strömt rou der Peripherie nach dem Centrum h i n. Beobachtet man diese Erscheinung bej $T$ ha lassicolla n u cleata nder noch besser bei Th. pelag ica, so kann man die einzehen Folgen dieses centripetalen Riticktliesseus des Protoplasmas nach eivander feststellen. Zunächst verkitreu sich die Pseudopodien und ziehen sich almäblich ganz ein, indem das Protoplasma sich in die Vactolenschicht zurickzieht. Dauert die Reizang noch länger, so zieht es sich auch vou hier immer mehr nach der Umgebung der Centralkapsel zuriick, wo es sich zu einer soliden schicht ansammelt. Die Folye dieses Riickzuges aus der Vacuolenschicht ist, dass ron der Peripherie her die Protoplasmawände der Vacuolen immer diinner und dimner werden, so dass die Vacuolen schliesshich phatzen. Dieses Vacuolemplatzen greift bei andanernder Reizung ron der Peripherie her immer mehr nach der Centralkapsel hin um sich, je weiter das Protoplasma sich centripetal zuriekzieht, bis schliesslich nur noch ein kleiner Rest unzerfallener Vacuolen ibrig ist. Danu berinnt die Thalassicollenzelle allmählich zu sinken, um so schneller, je geringer dic Zahl der noch vorhandenen Vacuolen wird. Hicr liegt also der Grund für das Sinken klar aut der Hand. Je uneh d ie Vacuolen, wolehe das specifisch leichteste Filement des Zellkirpers, gewissermassen seine bydrostatischen Apparate sind, zerplatzen, un so schwerer wird der Kirper, bis er schliesslich zu Boden siukt.

Ist die Thalassicollen zelle in Folge des Zerplatzens ihrer Vacuolen in die Tiefe gesunken, so ist es nach den im Vorstehenden festgestellten Thatsachen klar, dass sie nur wieder in die Höhe steigen kanndurch Regeneration ihrer Vacuolenschicht, die ja dasjenige Element des Kirpers repräsentirt, welohes specifisch leichter als das nugehende Meerwasser ist. 
Da die Entstehnngsweise der Vacunlen für die Frage, wie es komint, dass der Vacuoleninhalt specifisch leichter istals das umgebende Meerwasser, von Wichtigkeit sein musste, so suchte ich dic Neubildung: der Vacuolen genau zu verfolgen. Zu diesem $Z$ weck isolirte ich auf die oben angegebene Weise Centralkapseln aus dem Körper. Diese Centralkapseln haben die Fähigkeit, sich in wenigen Tagen zu vollkommenen Thalassicollen mit Pseudopodien, Gallertschicht und Vacuolen zu regeneriren, eine Lrscheinung, die nichts auffallendes hat, wenn man berïcksichtigt, dass der Inhalt der Centralkapsel den Werth einer vollständigen Zelle hesitzt, dass er aus Protoplasma und Zellkern bestelit. Ueberlässt man die isolirten Centralkapseln in einem Schälchen mit frischem Meerwasser sich selbst, so bemerkt man, dass bereits ganz kurze Zeit, häufig nur wenige Minuten nach der Isolation ringsherum an der ganzen Oberfläche feine Spitzchen hervortreten, die vorne Knöpfchen bekommen und sich immer mehr verlängern, bis sie sich zu einem dichten, radiär um den Körper angeordneten Kranz von Pseudopodienfäden ausgezogen haben. Diese Pseudopodien scheiden, wenn sie eine gewisse Länge erreicht haben, jedes an der ganzen Länge seiner Oberfläche, ein feines, schleimiges Secret ans, eine Erscheinung, die besonders nach Reizung durch Erschütterung sehr deutlich zu beobachten ist. Die Schleimhiillen der einzelnen Pseudopodien verkleben untereinander und schliesslich ist die Oberfläche der Centralkapsel von einer zusammenhängenden Schleimschicht überzogen, durch welche die Pseudopodien radiär hindurch frei ins Medium hinausragen. Nachdem die Schleimschicht eine gewisse Dicke erreicht hat, bemerkt man in ihr zwischen den Pseudopodiensträngen äusserst kleine, blasse Wassertröpfchen hier und dort vereinzelt auftreten. Diese Wassertröpfchen vergrössern sich durch Aufnahme von mehr Wasser aus dem Medium her, verschmelzen untereinander und werden immer deutlicher, indem sie ringsherum von dem auseinander gedrängten Protoplasma der dicht stehenden Pseudopodien umbüllt erscheinen. Das Protoplasma der Pseudopodien liefert bald eine ringsherum gesehlossene Wand für diese primitiven kleinen Vacuolen. D i e s o von einer $\mathrm{Pr}$ toplasmawand umgebenen Fli ssigkeits racuolen fabren immer weiter fort, sieb dureh Wasseraufnahme vom Medium ber zu vergrösern, bis sie oft schon nacheinem halben TagezuVacuo- 
Ueber die Fähigkeit der Zelle, activ ihr specifisches Gewicht zu verändern. 151

len von normalerGrösse herangewachsensind. Durch Fortschreiten des eben beschriebenen Processes vermehrt sich die Zahl der Vacuolen nach und nach und die Vacuolenschicht nimmt immer mehr an Mächtigkeit zu. Hebt man dann die $\mathrm{T}$ balassicolla in Wasser an die Oberfläche und laisst sie los, so bemerkt man, dass sie jetzt schon bedentend langsamer in Wasser wieder untersinkt als die frisch isolirten Centralkapseln, die noch keine Vacuolenschicht regenerirt haben, und nach wehreren Tagen kann die Vacuolenschicht bereits eineu solchen Unfang angenommen haben, dass die $\mathrm{T}$ h a $\mathrm{l}$ a s s i c o l l e u ze l l e langsam sich vom Boden des Gefässes zu erheben und im Wasser in die Höhe zu steigen beginut, bis sie dauernd an der Oberfläche verharrt.

Die vorstehenden Versuche zeigen, dass die Vacuolenschicht der hydrostatische Apparat der Radiolarienzelle ist. Die Vacuolenfliissigkeit ist derjenige Theil der Zelle, welcher specitisch leichter als das Meerwasser ist und die Zelle daher an der Obertliiche schwebend erhält. Durch andauerude Reizung zerplatzen die Protoplasmawände der Vacuolen, die Flüssigkeit tritt heraus und bei einer genügenden Verminderung der Zahl der Vacuolen wird die Zelle schwerer wic Meerwasser und fängt an zu sinken. Durch Regeneration der Vacuolen dagegen steigt die Zelle wieder in die Höhe.

Es fragte sich nun, ob sich auch bei den Ctenophoren Anhaltspunkte dafür finden lassen, dass das Steigen und Sinken, soweit es nicht durch active Bewegung der Locomotionsorgane bedingt ist, aut älnlichen Verbältnissen beruht.

Es ist bekanut, dass der Körper der C'tenophoren melr als $96 \%$ Wasser entuält, dass also die Menge der festen Sulstanz gregenüber dem Wasser verschwindend gering ist, ein Unstand, der schon gecignet ist, ein sehr viedriges specifisches Gewicht zu bewirken. Betrachtet man den Körper der Ctenophoren histologisch, so findet man, dass die Zellen fast aller Gewebe stark racuolisirt sind und dass der Protoplasmakörper manchmal wie bei Pflanzenzellen nur dünne Protoplasmastränge und Protoplasmawände durch die Vacuolenflüsigkeit sendet. Durch cinen Blick auf die ausge- 
zeichneten Abbildungen $\mathrm{Ch} \mathrm{un} \mathrm{s}^{\mathbf{1}}$ ) in seiner Monographie der Ctenophoren kann man sich jederzeit von dieser Thatsache iiberzeugen. Nun können die Ctenophoren bei ibrer Entwicklung die Vacuolenflitssigkeit nur aus dem umgebenden Meerwasser beziehen, denn die Eizellen and die ersten Entwicklungsstadien der Ctenophoren repräsentiren noch ziemlich solide Protoplasmamassen. Es liegt also hier ein analoges Verhältniss vor wie bei den Radiolarien.

Auch für das Sinken finden sich Analogieen. Es ist eine eigenthümliche Erscheinung, dass alle pelagischen Thiere, die man in der Gefangenschaft hält, ganz enorm an Volumen abnehmen, ohne dabei ibre Form oder ibre Lebensthätigkeiten zu verändern. Eine Beroë Forskalii, die ich in einem grossen Wasserbassin hielt, war kurz nach dem Einfangen $2 \mathrm{~cm}$ gross; nach 10 'Tagen betrug ihre Länge nur noch $6 \mathrm{~mm}$, sie war also nahezu auf den 4. Theil ihrer Grösse zusammengeschrumpft, obne dabei die geringste Störung in ihrem sonstigen Verhalten zu zeigen. Ich fand nun, dass alle diese Thiere, welche geschrumpft waren, selbst wenn sie vorher specifisch leichter waren als das Meerwasser, zu Boden sanken oder am Boden lagen, sobald sie ibre Locomotionsorgane nicht benutzten, dass sie also mit dem Schrumpfen specifisch schwerer geworden waren als das Meerwasser. Da das Schrumpfen wesentlich nur auf einer Verminderung des Volumens der Vacuolenflüssigkeit beruht, so liegt es nahe, dass auch hier die Vacuolenfluissigkeit dasjenige Element ist, das specifisch leichter ist als Meerwasser und durch dessen Volumenverminderung oder -Vermehrung das Sinken oder Steigen bedingt ist. Auch absterbende Ctenophoren, bei deren Gewebezellen die Vacuolenwände einen Austausch der immeren Flïssigkeit mit dem umgebenden Meerwasser gestatten, sind immer specifisch schwerer wie Meerwasser und sinken darin zu Boden.

WaLrscheinlich werden sich ähnliche Verhältnisse noch bei vielen anderen pelagischen Thieren finden, für die ja alle ein niedriges specifisches Gewicht von grosser biologischer Bedeutung ist und deren Gewebezellen ebenfalls einen stark vacuolisirten Charakter haben.

1) Chun, „Die Ctenophoren des Golfes von Neapel und der angrenzenden Meeresabschnitte." Leipzig 1880. 
Ueber die Fähigkeit der Zelle, activ ihr specifisches Gewicht zu verändern. 153

\section{Der Mechanismus des Steigens und Sinkens.}

Nach den oben beschriebenen Versuchen fehlt in dem Gesammtbild des Mechanismus, welcher das Steigen und Sinken der pelagischen Thiere vermittelt, noch ein Momeut. Die Versuche zeigen, dass mit der Entwicklung und Vergrössermug der Vacuolen das specifische Gewicht der Zelle sich vermindert, dass die Vacuolenfliissigkeit also specitisch leichter wird als das Meerwasser. Die Frage, die noch zu beantworten ibbrig bleibt, ist daher die: Wie kommt es, dass die Vacuolenflits sigkeit specifisch leichter ist als das umgebende Meerwasser a us dem sie stammt? Un diese Frage zu entscheiden sind keine neuen Versuche mehr nöthig. es bedarf nur der Erimernug an eine längst allgemein bekannte und besonders von den Ptlanzenphysiologen eingehend studirte Thatsache.

Jedem Histologen, der lebendige Objecte unter dem Mikroskop untersucht hat, ist die Erscheinung entgegengetruten, dass die lebendige Zelle, solange ihre Lebensvorgänge noch ungestört fortbestehen, Farbstofte, die sich in Lisung betinden, in der Regel nicht in ihr Inneres aufnimmt. Setzt man z. B. zn einem Wassertropfen, in dem sich einzellige Organismen, etwa Amoeben oder Infusorien befinden, oder auch zu eiuem Stäck ibbrhbenden Gewehes von einem höheren Thier eine jener Farlostofflösungen, die für herufarbngyeu benutat werdeu, so sieht man, dass selbst im Verlauf längerer Zeit eine Färbung der Zellen nicht eintritt und dass die Zellen, sofern die in der larbstoffliosung enthaltene säure oder der Alkohol sie nicht zum Alsterben bringt, weiter leben, ohne den Farbstoff' in sich antzunehmen. In der dunkelrothen Lösung" eines Karminfarbstoffes sieht man unter gecigneten Bedingungen die Zellen noch stumdenlang die blassgrane Farbe ihres Protoplasmas erhalten. Erst in dem Maasse, wie die Zelle vor der Peripherie her abstirbt, heginnt sich auch das Protoplasma mit dem Farbstoff an iubibiren. Der Histologe rechnet tïglich melnr oder weniger bewusst wit diesel Thatsache, denn er töltet stets vor der Fïrbung die Objecte erst ab. Dic lebendige Substanz der Zelle lässt also, solange ihre Lehensvorgänge ungestiort sind, den Farbstoff nicht in sich eindriugen.

Das wleiche Verhalten zeint sich Zucker- und Salzlïsungen gegenüber, eine Erscheinung, die von pflanzcnphysiologischer seite 
bis in die feinsten Einzelheiten genau untersucht worden ist. Bekanntlich wird die Cellulosekapsel einer lebendigen Pflanzenzelle an ihrer Innenfläche von einer feinen, änssert zarten, zusammenhängenden Protoplasmahaut, dem sog. Primordialschlauch tiberzogen. Der Primordialschlauch stellt einen Sack vor, der den Zellsaft umhüllt, durch den er nach den verschiedensten Richtungen Protoplasmastränge entsendet. Wird eine Pflanzenzelle in eine schwache Zucker- oder Salzlösung gelegt, von einer Concentration, welche der lebendigen Substanz nicht schädlich ist, so tritt eine „Plasmolyse" ein, d. h. der Primordialschlauch, hebt sich von der Cellulosewand, der er vorher dicht anlag, ab und zieht sich auf eine geringere Oberfläche zusammen. Das beruht darauf, dass die im Medium gelösten Zucker- oder Salzmoleküle nicht durch den Primordialschlauch in den Zellsaft hinein diffundiren können. In Folge dessen entziehen sie dem Zellsaft eine gewisse Menge Flüssigkeit, der Zellsaft nimmt an Volumen ab und der Turgor der Zelle sinkt bis zu dem Punkte, wo die Turgorkraft der wasseranziehenden Kraft der Zucker- oder Salzmoleküle wieder das Gleichgerwicht bält. De Vries ${ }^{1}$ ) hat diese plasmolytische Methode benutzt, um in genialer Weise die Grösse der Turgorkraft, d. b. der Kraft, mit welcher das Wasser von der lebendigen Zelle festgehalten wird, zu bestimmen. De Vries fand ferner, dass bei langsamem Absterben der Zelle das Protoplasma zuerst für leicht diffusible, später auch für schwer diffusible Stoffe durchgängig wird. Dabei nimmt die Zelle, wenn sie vorher plasmolytisch war, wieder mehr Wasser auf, ihre Vacuolen werden grösser und der Primordialschlauch dehnt sich wieder aus. D a s P o toplasma ist also impermeabel für diebetreffenden Stoffe, solangeesin ungestörtlebendigem $\mathrm{Zustande}$ is t.

In dieser allgemein bekannten Impermeabilität des lebendigen Protoplasmas für gewisse in Lösung befindliche Stoffe liegt nun, wie leicht einzusehen ist, auch die Ursache für das geringere specifische Gewicht der Vacuolen bei den pelagischen Seethieren. Wenn

1) De Vries, „Eine Methodè zur Analyse der Turgorkraft." In Pringsheim's Jahrbücher für wiss. Bot. Bd. XIV. 1884. — „Plasmolytische Studien über die Wand der Vacuolen." In Pringsheim's Jahrbücher f. wiss. Bot. Bd. XVI. 1885. 
Ueber die Fähigkeit der Zelle, activ ihr specifisches Gewicht zu verändern. 155

man die Art und Weise der Bildung and des Wachsthums der Vacuolen ins Ange fasst, so hat man hier dieselben Verhältnisse wie bei der Pflanzenzelle. Die Vacuolen der Radiolarien treten als kleine mikroskopische Bläschen auf, die von einer Protoplasmawand umgeben sind. Sie wachsen, indem sie rom Medium ber Wasser in sich aufnehmen. Dabei muss das Wasser die protoplasmatische Vacuolenwand passiren. Ist aber das lebendige Protoplasma für Salze undurehgängig, so können die in dem Meerwasser enthaltenen Salze nicht mit hindurchtreten. Es gelangt also eine salzfreiere Fliissigkeit in die Vactolen, die natürlich specifisch leichter sein muss als das salzhaltige Hcerwasser. Wieriel diese Differenzen im specifischen Gewicht des Meer- und des Siisswassers ausmachen, weiss jeder, der im Heer und in Landseen öfters gebadet hat. Im Landsee ist der mensehliche Körper viel schwerer als im Meer, und es bedarf im Meer kaum irgend welcher Anstrengungen, um sich an der Oberflïche zu erhalten. So wird es verständlich wie die Radiolarien\%elle, wenn die Vacuoleubildung einen gewissen Grad erreicht hat, specitisch leichter wird, als das ungebende Meerwasser, so dass die Zelle in die Höbe steigt. Fängt die Protoplasmawand der Vacuolen an abzusterben, so dass sie durchlüssig für die Salze des Meerwassers wird, so gleicht sich die Gewichtsvermindermng wieder aus und die Zelle sinkt in Folge der Schwere ihrer eigenen substanz langsam tiefer. Dasselbe tritt ein, wenn die Vacnolen in Folge von Reiznngr ganz zerplatzen.

Diejenigen Krïfte, welche das Wasser vom Medium her in die Radiolarienzelle hineinzieben, sind natiulich dieselben wie bei jeder anderen Vacuolenbildung, d. b. die osmotischen Kräfte, welche auch den Turgor der Pflanzenzelle bedingen. Man muss dabei ror. aussetzen, dass die im Protoplasma enthaltenen osmotisch wirksamen Moleküle diejenigen des Wassers stärker oder in grösserer Menge anziehen als die Salzmolekiile des Seewassers selbst cs thun, d. h. um De V ries Ausdrueksweise zu grebrauchen, dass ihr ,isotonischer Cö̈fficient" ein grö̈sserer ist. Zur nähẹren Kenntniss dieser Verhältnisse muss bier anf die ausgezeichneten Arbeiten von P felfer und De Vries verwiesen werden.

Hiermit ist ein liickenloses Bild ron dem Mechanismus gewonnen, der das geheimnissvolle Steigen und Sinken jener wunderbaren pelagischen $W$ esen rermittelt, die in ihrcl geisterhaften Durchsichtigkeit ohue die geringste Bewegung ihres ätherischen Körpers auf- und niederschwebend ein märchenhaftes Dasein fiihren. 